\title{
Building Communities with People-Tags
}

\author{
Stephen Farrell, Tessa Lau, and Stefan Nusser \\ IBM Almaden Research Center \\ San Jose CA 95120, USA \\ sfarrell@almaden.ibm.com
}

\begin{abstract}
Social tagging has been applied to many applications including image sharing, bookmarking and music recommendations. We have developed a application for the social tagging of people to support contact management and browsing profiles in an enterprise directory. As we expected, we found that users tag people for personal organization and for "social" motivations just as in other systems. However, an unexpected result is that users tag other users in order to create communities. By tagging, encouraging others to tag, and sharing links to tags, an active minority of users is using people-tagging to bring people together.
\end{abstract}

\section{Introduction}

Social tagging has been applied widely to enrich applications for sharing bookmarks, photos, videos, music, podcasts and other resources. We have built a social tagging application for enterprise contact management called "Fringe Contacts" which enables employees to place themselves and their fellow employees in a folksonomy of skills, interests and projects [4.

More recently, we have integrated social tagging into our enhanced directory prototype "Fringe". Through usage statistics, surveys and interviews, we learned that tagging is useful for personal organization, enables an active minority to contribute useful and accurate information to other's profiles, and that tag frequency provides a basis for ranking people. Moreover, early evidence suggested that users policed themselves and that inappropriate and undesired tags were rare in this environment $[5]$.

An unexpected result that emerged from our interviews, which was not reported in previous work, is that users frequently tag people for the benefit of others. They wanted users to find out about each other and to encourage them to start using the tagging feature. They included snapshots of tag groups in presentations, they shared links to tag-based groups of people, and they used tags to construct ad hoc mailing lists. In short, they used people-tagging to define and socially manage communities. In this paper, we report on this result and speculate on what it means for the design of future tagging communities.

\section{Related Work}

Social bookmarking systems enable users to tag web pages and contribute to an emergent folksonomy of web pages [1] 2]. Social tagging has also been successfully 
applied to other media including images (flickr.com), videos (youtube.com) and music (last.fm). Marlow et al surveyed tagging systems and have developed a taxonomy of system design parameters and user incentives. They have found tags used for future retrieval, contribution and sharing, attracting attention, play and competition, self presentation, and opinion expression [3].

\section{Interviews}

We have found a substantial number of users - 3,726, more than $1 \%$ of all employees - contributing tags to our system. We asked a number of the top users to participate in a 30 minute interview. We selected these users based on the number of people who had tagged them, the number of people they had tagged, and the duration of time they have been using the system. In the end, we conducted 19 semi-structured interviews.

\section{Results}

We found that many of the same incentives that applied to other social tagging systems also applied to person-tagging. Most respondents reported using tags to organize their contacts to help with future retrieval. Most reported clicking on a tag to recall people, and about half mentioned that they found it useful to see their own tags while browsing others' profiles, reminding them what they knew of the other person.

We were surprised to learn how many respondents reported using tags for contribution and sharing. 15 of the 19 respondents described sharing (or interest in sharing) tags to inform others about a group or to inform members of the group about each other. This was not necessarily altruistic behavior: many respondents had an interest in the success of the community and found people-tagging a means to that end.

Community builders were resourceful in finding ways to leverage person-tags. One respondent tagged participants in a program he was running tap-innovator so he could demonstrate to his management the range of people involved. Another lead a cross-organizational study on patterns in software development and tagged its members by subtopic and embedded links to these groups in the project wiki. Others shared tags by directing people to their profile, emailing links, or even including screen-shots in presentations. Two respondents had not thought of sharing tags, but, upon the suggestion, wanted to try it. Some of these respondents reported using the list of email addresses that appears on the tag page as an ad hoc mailing list.

Some community builders have taken person-tagging to the next level. Realizing that users could tag themselves or anyone else, they enlisted the community to manage itself. One respondent described her practice of sharing links and encouraging reuse of tags as "helping the community to self-organize".

We learned about the first example of self-organizing communities prior to our interviews with the chi2006 tag. One user had tagged everyone he knew that 
was attending the $\mathrm{CHI}$ conference that year. He then sent them an email that included a link to the group and instructions to tag anyone who was missing. In the end, a comprehensive list of everyone attending the conference had been produced by a few people in an unstructured way. This behavior has recurred with web20forbiz, p-vista and, recently, chi2007.

One of our respondents described how he used person-tagging to help build a community around the virtual reality program Second Life. He described an early practice of asking people in email, web pages and presentations to tag themselves "secondlife" so they could find each other. However, he backed off of this practice when the community reached into the thousands.

In other cases, this application of tagging failed to reach critical mass. One respondent commented on a tag that had only been used twice: "Bob is trying to get that going". Since taggees are not notified upon being tagged, the ability for tags to spread virally is limited. All of the successful examples we know of have had champions using other channels to encourage the spread the use of the tag.

We also uncovered some interactions with an existing system for managing groups called "Bluegroups". One respondent started creating a community with a tag but then switched to Bluegroups so he could use the membership list for access control. Conversely, another respondent translates the names of Bluegroups that appear on users' profiles into tags. Yet another respondent manually synchronizes the two. A final respondent felt that a Bluegroup was too large to be useful to him, so he tagged just the "key people" in the group.

We found users took advantage of the flexibility of the semantics of person tagging in community building. One respondent explained the tag webdevlounge: "we use this tag to organize everything". Indeed, they also use it for social bookmarks, blogs, wikis, and other tools. For this community, tagging a person webdevlounge in Fringe means that the person is of interest to the team, not a member. A Bluegroup serves the purpose of managing team membership.

An interesting variant of community tagging, perhaps related to play and competition, was shaming. One respondent wanted to encourage his colleagues to upload photos to their profile. He tagged them no-picture-in-bluepages and removed the tag once this had been rectified. There is no one with this tag now.

We also found examples of other incentives enumerated by Marlow including attracting attention and self presentation. Users tag themselves with their skills and projects, in effect enabling others to find them by browsing and influencing the tags that appear on their profiles. We also found tags used for play and competition. For example, we found the use of sassy and needs-a-shave. We found that respondents did not use these tags to describe themselves suggesting that they serve a social rather than informative role. We also found this result with affirmative tags like organized and rockstar.

Finally, we found evidence of disincentives to tagging people. Respondents withheld tags due to presumed consequences. One respondent reported that he did not tag a colleague with the name of a former project because he knew 
the colleague did not want to be contacted about it. While some respondents suggested it could be useful, all were reluctant to use people-tags for opinion expression. One respondent put it: "I try to be very conservative, and not make judgments and aspersions about them.... I try to use this as a very positive thing."

\section{Conclusion}

We have found that people-tagging as implemented in Fringe fits well into the family of other tagging systems as outlined by Marlow et al. The same basic motivations of self-interest and "social" contribution play major roles.

We think creating community, while related to contribution and sharing, is itself a distinct incentive. Users already motivated to create communities have found people-tagging a useful tool to demonstrate the community to others and introduce the members to each other. Moreover, they have leveraged the openness of tagging to enable the community to maintain itself.

We expect to find the incentive of creating community in other tagging applications. For example, someone might use the tag "sanfrancisco" on flickr not only to associate the photo with a topic area, but also to help photographers in San Francisco find each other.

In the future, we would like to study how these tag-defined communities compare with opt-in or administered communities on Facebook and other social network sites. We are also looking at extending email and instant messaging tools to enable users to contact people by tag. We may also introduce features to help "viral" spreading of tags by enabling users to notify others of the tags as they assign them. As person-tags are more widely used and the tools to leverage them become more powerful, we will continue to watch user's reactions to being tagged and report on our findings.

\section{References}

1. Golder, S., Huberman, B.A.: Usage patterns of collaborative tagging systems. Journal of Information Science 32(2), 198-208 (2006)

2. Millen, D.R., Feinberg, J., Kerr, B.: Dogear: Social bookmarking in the enterprise. In: CHI '06. Proceedings of the SIGCHI conference on Human Factors in computing systems, pp. 111-120. ACM Press, New York (2006)

3. Marlow, C., Naaman, M., Boyd, D., Davis, M.: HT06, Tagging Paper, Taxonomy, Flickr, Academic Article, ToRead. In: HT '06. Proceedings of Hypertext, ACM Press, New York (2006)

4. Farrell, S., Lau, T.: Fringe contacts: People-tagging for the enterprise. In: Workshop on Collaborative Web Tagging, WWW 2006 (2006)

5. Farrell, S., Lau, T., Wilcox, E., Nusser, S., Muller, M.: Socially augmenting employee profiles with people-tagging. Submitted to UIST (2007) 